\title{
PLANT GROWTH, METABOLISM AND ADAPTATION IN RELATION TO STRESS CONDITIONS: \\ XXVIII. PHYSIOLOGICAL EFFECTS OF UV RADIATION ON GROWTH AND PHOTOSYNTHETIC CAPACITY OF GERMINATING BROAD BEANS.
}

Younis, M. E; M. N. A. Hasaneen and Heba M. Abd-elAziz. Department of Botany, Faculty of Science, Mansoura University, Mansoura, Egypt.

\begin{abstract}
Exposure of broad bean seedlings either germinated in dark conditions and/or in light conditions (40 W, low intensity and $160 \mathrm{~W}$; high intensity) during germination to UV-A $365 \mathrm{~nm}$ and UV-C $254 \mathrm{~nm}$ for one hour daily throughout the entire period of the experiment (six days), led to significant decrease in all growth parameters determined (length of radicle, length of plumule, water content, fresh weight and dry weight) as compared with control seedlings. Significant changes were observed in the amount and in the relative composition of photosynthetic parameters (Chl a, Chl b, Chl a+b, Chl a/b, Cars, Total pigments) of the variously treated broad beans in relation to control samples. Photosynthetic activity expressed in the present work as the reduction of 2,6-DCPIP (PS II activity) of the differently treated broad beans showed variable significant changes as compared with control seedlings throughout the entire period of the experiment. These results are discussed mainly on the basis of the mechanism of action of UV radiation on growth and metabolic changes in broad beans during germination in dark or in light conditions.

Keywords: UV-radiation, germination, growth parameters, photosynthetic pigments, photosystem II activity, Vicia faba L.
\end{abstract}

\section{INTRODUCTION}

Ultraviolet radiation (UVR) makes up about $8 \%$ of solar irradiance reaching the earth. Most UV-radiations are screened out by the ozone layer and the intensity of UV-radiations is affected by the thickness of the ozone layer. Ozone layer is being depleted as a result of contamination with manmade ozone depleting substances (ODS) (Farman et al., 1985; Jordan, 1996; Saleh et al., 2006)

The direct effects of ultraviolet radiations on plant cells are mostly damaging, because UV photons have enough energy to create lesions in important UV-absorbing biomolecules such as nucleic acids and proteins (Taylor et al., 1997). In the leaves of tropical trees, the ambient UV-B and UV-A radiation might contribute to the reversible decline in potential photosystem II (PS II) efficiency observed upon exposure to full, direct sunlight. Increased levels of UV-absorbing compounds and protein damage were indicated by strong effect of photosynthetically active radiations (PAR/ UV light) (Krause et al., 1999; Saleh et al., 2006).

Saradhi et al. (1995) suggested that exposure of rice plants to ultraviolet radiation reduces plant growth vigor, chlorophyll contents, 
carotenoids, total sugars and starch, but increases the level of anthocyanin and proline (Musil,1996). Ultraviolet light inhibited the growth in four wheat cultivars (Triticum aesitivum L.) and increased proline contents which were thought to protect cells against damage (Demir, 2000). Todorov et al. (2003) found that UV-irradiation decreased fresh and dry weight of plants and increased the free proline content during UV-B and UV-C stress and recovery period.

Thus the objective of this study was to investigate the effects of different ultraviolet radiation (UV-A and UV-C) doses on growth and photosynthetic capacity of broad bean seedlings (Vicia faba L. c.v. Egypt 1) germinated either in dark or in light conditions throughout the entire period of the experiment.

\section{MATERIALS AND METHODS}

\section{Plant material and germination:}

Broad bean (Vicia faba L. cv. Egypt 1) seeds of similar size and appearance were selected. Seeds were sterilized with $2.5 \%$ sodium hypochlorite solution for $15 \mathrm{~min}$. and washed with distilled water. These seeds were then germinated in plastic boxes $(25 \mathrm{~cm}$ in length $\times 10 \mathrm{~cm}$ in width) on Whatman No. 1 filter paper equally watered with $25 \mathrm{ml}$ of distilled water. All boxes were divided into two groups, one of them left for germination in the dark and the other left for germination in normal light for 14 days until UV and light-treatments.

Irradiation system and germination conditions:

After 14 days from sowing, seedlings of the first group (dark group) were divided into six subgroups each of 4 boxes, one of them was left in dark as control and the other five subgroups were treated as follows, 1- Exposed to low light intensity $(2731.9 \mathrm{~K} \mathrm{Lux})$ for $1 \mathrm{~h} /$ day every 2 days interval for 6 days,2- Exposed to short UV (452 nm) for $1 \mathrm{~h} /$ day every 2 days interval for 6 days,3- Exposed to low light intensity (2731.9 K Lux) in combination with short UV $(452 \mathrm{~nm})$ for $1 \mathrm{~h} /$ day every 2 days interval for 6 days, 4- Exposed to long UV $(365 \mathrm{~nm})$ for $1 \mathrm{~h} /$ day every 2 days interval for 6 days and 5- Exposed to low light intensity (2731.9 K Lux) in combination with long UV (365 nm) for $1 \mathrm{~h} /$ day every 2 days interval for 6 days.

Seedlings of the second group (normal light group) were subdivided into 4 subgroups; one of them was left in normal light conditions as control and the other three subgroups were treated as follows, 1- Exposed to high light intensity (10927.9 K Lux) for 1h/ day every2 days interval for 6 days,2Exposed to short UV (452 nm) for $1 \mathrm{~h} /$ day every 2 days interval for 6 days and 3- Exposed to high light intensity (10927.9 K Lux) in combination with short UV $(452 \mathrm{~nm})$ for $1 \mathrm{~h} /$ day every 2 days interval for 6 days. Irradiation treatments were applied using light boxes contains UV lamps (365 nm, 254 $\mathrm{nm}$ ) and fluorescent lamps (2731.9 K Lux, 10927.9 K Lux). Timers were set to automatically turn irradiation Lampson at midday time as possible. Radiation doses and radiation power emitted from UV lamps were calculated according to distance between lamp axis and broad beans as presented in the lamp instruction manual (Gilbert, 1996). Distance between lamps and 
upper leaves of broad bean were set to $45 \mathrm{~cm}$ and were periodically monitored and reset as broad bean grows (Saleh et al., 2006).

Growth parameters:

Length of root, length of shoot, fresh weight, dry weight and water content were estimated before and after treatment.

\section{Determination of pigments:}

Photosynthetic pigments ( $\mathrm{Chl} \mathrm{a,} \mathrm{Chl} \mathrm{b}$ and carotenoids) were determined using the spectrophotometric method as described by Metzner et al. (1965). A known fresh weight of seedlings was homogenized in $85 \%$ aqueous acetone for $5 \mathrm{~min}$. The homogenate was suction filtered through Whatman No. 1 paper. The filtered extract was made up to volume with $85 \%$ aqueous acetone. The extract was measured against a blank of pure $85 \%$ aqueous acetone at three wavelengths of $452.5,644$ and $633 \mathrm{~nm}$ using a Spekol spectrocolourimeter.

Hill reaction assay:

As described by Arnon (1949), leaf discs were used for preparation of chloroplast pellets that were suspended in $1 \mathrm{mM}$ Tricine- $\mathrm{NaOH}(\mathrm{pH} 7.8), 10$ $\mathrm{mM} \mathrm{NaCl}$ and $10 \mathrm{mM} \mathrm{MgCl}_{2}$ and then kept at $0-4^{\circ} \mathrm{C}$ until required. PS II activity, as indicated be the rate of 2,6 dichlorophenol indophenol (DCPIP) photoreduction was monitored at $606 \mathrm{~nm}$ using a Spekol spectrocolourimeter.

The data of the different treatments were statistically analyzed using the test of the least significant difference (L.S.D.) at $5 \%$ level (Snedecor and Cochron, 1980).

\section{RESULTS AND DISCUSSION}

\section{Changes in growth parameters:}

The results depicted in tables (1-10) show that there was a steady increase in all growth parameters (length of radicle, length of plumule, fresh mass, dry mass and water content) measured in broad bean seedlings exposed to white light and/ or UV-A and UV-C radiations either alone or in combination in relation to control seedlings germinated either in dark or in normal light conditions.

Exposure of dark-germinated or light-germinated broad bean seedlings to low light, high light, UV-A, UV-C either alone or in combination involved significant variable decrease in all growth parameters measured, as compared with those growth parameters measured in dark-germinated or light-germinated control seedlings throughout the entire period of the experiment.

Because plants must be exposed to sunlight to power photosynthesis, they are exposed to high levels of UV-A and UV-C radiations in the biosphere which might damage the performance of many crop plants (Saleh et al., 2006). In the present study, UV-A and UV-C radiations either alone or in combination with low and high light intensities, reduced all growth parameters of germinating broad beans, may be attributed to photosynthetic mechanisms. Plant photosynthetic UV-A and UV-C effects may be associated with changes in cell division and/ or cell elongation (Gehrke.1999; Caldwell et al., 2003; Saleh et al., 2006). 
Younis, M. E. et al.

Table1: The effect of low light and UV radiations either alone or in combination on the length of radicle $\left(\mathrm{cm}^{\text {seedling }}{ }^{-1}\right)$ of Vicia faba seedlings germinated under dark conditions. Mean values are significantly different from control at ${ }^{*} P \leq 0.05$.

\begin{tabular}{|c|c|c|c|c|}
\hline \multirow{2}{*}{\multicolumn{2}{|c|}{\begin{tabular}{|cc} 
Treatment & Day \\
Control (dark) &
\end{tabular}}} & 2nd & 4th & 6th \\
\hline & & 4.50 & 4.70 & 4.90 \\
\hline \multirow{3}{*}{ Low light } & Before & 4.43 & $4.46^{*}$ & $4.60^{\star}$ \\
\hline & After & 4.40 & $4.45^{*}$ & $4.55^{*}$ \\
\hline & Difference & 0.03 & 0.01 & 0.05 \\
\hline \multirow{3}{*}{ Short UV } & Before & 4.45 & 4.60 & 4.73 \\
\hline & After & 4.43 & 4.50 & $4.65^{\star}$ \\
\hline & Difference & 0.02 & 0.1 & 0.08 \\
\hline \multirow{3}{*}{$\begin{array}{l}\text { Low light + } \\
\text { Short UV }\end{array}$} & Before & 4.32 & $4.40^{*}$ & $4.45^{*}$ \\
\hline & After & $4.26^{*}$ & $4.30^{*}$ & $4.35^{*}$ \\
\hline & Difference & 0.06 & 0.1 & 0.1 \\
\hline \multirow{3}{*}{ High UV } & Before & 4.70 & 4.82 & 4.85 \\
\hline & After & 4.63 & 4.80 & 4.84 \\
\hline & Difference & 0.07 & 0.02 & 0.01 \\
\hline \multirow{3}{*}{$\begin{array}{c}\text { Low light + } \\
\text { High UV }\end{array}$} & Before & $4.35^{\star}$ & 4.55 & $4.56^{*}$ \\
\hline & After & $4.33^{*}$ & 4.50 & $4.53^{*}$ \\
\hline & Difference & 0.02 & 0.05 & 0.03 \\
\hline \multicolumn{2}{|c|}{ L.S.D at ${ }^{*} P \leq 0.05$} & 0.20 & 0.20 & 0.21 \\
\hline
\end{tabular}

Table2: The effect of low light and UV radiations either alone or in combination on the length of plumule $\left(\mathrm{cm}\right.$ seedling $\left.{ }^{-1}\right)$ of Vicia faba seedlings germinated under dark conditions. Mean values are significantly different from control at ${ }^{*} P \leq 0.05$

\begin{tabular}{|c|c|c|c|c|}
\hline Treatment & Day & 2nd & 4th & 6th \\
\hline Contrc & ark) & 5.20 & 5.73 & 6.80 \\
\hline & Before & $4.30^{*}$ & $4.40^{*}$ & $4.60^{*}$ \\
\hline Low light & After & $4.20^{*}$ & $4.30^{*}$ & $4.50^{*}$ \\
\hline & Difference & 0.1 & 0.1 & 0.1 \\
\hline & Before & 5.20 & $5.40^{*}$ & $5.65^{\star}$ \\
\hline Short UV & After & 5.00 & $5.30^{\star}$ & $5.50^{*}$ \\
\hline & Difference & 0.2 & 0.1 & 0.15 \\
\hline & Before & 5.15 & $5.24^{*}$ & $5.37^{*}$ \\
\hline Low ingt + snort & After & 5.10 & $5.19^{*}$ & $5.26^{\star}$ \\
\hline & Difference & 0.05 & 0.05 & 0.11 \\
\hline & Before & 5.18 & $5.30^{*}$ & $5.85^{\star}$ \\
\hline High UV & After & 5.16 & $5.20^{*}$ & $5.65^{\star}$ \\
\hline & Difference & 0.02 & 0.1 & 0.2 \\
\hline & Before & $4.80^{*}$ & $4.90^{*}$ & $5.12^{*}$ \\
\hline Low light + High & After & $4.70^{*}$ & $4.80^{*}$ & $4.92^{*}$ \\
\hline & Difference & 0.1 & 0.1 & 0.2 \\
\hline L.S.D at & $\leq 0.05$ & 0.26 & 0.28 & 0.34 \\
\hline
\end{tabular}


Table 3: The effect of low light and UV radiations either alone or in combination on fresh mass ( $g$ seedling ${ }^{-1}$ ) of Vicia faba seedlings germinated under dark conditions. Mean values are significantly different from control at ${ }^{*} \mathrm{P} \leq \mathbf{0 . 0 5}$.

\begin{tabular}{|c|c|c|c|c|}
\hline Treatment & Day & 2nd & 4th & 6th \\
\hline Cont & lark) & 2.55 & 2.66 & 2.74 \\
\hline & Before & $2.40^{\star}$ & $2.46^{*}$ & $2.62^{*}$ \\
\hline Low light & After & $2.39^{*}$ & $2.44^{*}$ & $2.59^{*}$ \\
\hline & Difference & 0.01 & 0.02 & 0.03 \\
\hline & Before & 2.57 & 2.65 & 2.69 \\
\hline Short UV & After & 2.50 & 2.60 & 2.64 \\
\hline & Difference & 0.07 & 0.05 & 0.05 \\
\hline & Before & 2.53 & 2.64 & 2.73 \\
\hline Low IIght + & After & $2.38^{*}$ & 2.55 & 2.68 \\
\hline & Difference & 0.15 & 0.09 & 0.05 \\
\hline & Before & 2.52 & 2.56 & 2.66 \\
\hline High UV & After & 2.43 & 2.55 & 2.65 \\
\hline & Difference & 0.09 & 0.01 & 0.01 \\
\hline & Before & $2.39^{*}$ & $2.43^{*}$ & $2.58^{*}$ \\
\hline Low light + High & After & $2.36^{*}$ & $2.41^{*}$ & $2.57^{*}$ \\
\hline & Difference & 0.03 & 0.02 & 0.01 \\
\hline L.S.D & $\leq 0.05$ & 0.12 & 0.13 & 0.13 \\
\hline
\end{tabular}

Table 4: The effect of low light and UV radiations either alone or in combination on dry mass ( $\mathrm{g} \mathrm{seedling}^{-1}$ ) of Vicia faba seedlings germinated under dark conditions. Mean values are significantly different from control at ${ }^{*} \mathrm{P} \leq 0.05$.

\begin{tabular}{|c|c|c|c|c|}
\hline Treatment & Day & 2nd & 4th & 6th \\
\hline Contrc & ark) & 0.60 & 0.62 & 0.64 \\
\hline & Before & $0.53^{*}$ & $0.54^{*}$ & 0.63 \\
\hline Low light & After & $0.50^{*}$ & 0.55 & $0.58^{*}$ \\
\hline & Difference & 0.03 & 0.05 & 0.05 \\
\hline & Before & 0.61 & 0.63 & 0.69 \\
\hline Short UV & After & 0.60 & 0.61 & 0.68 \\
\hline & Difference & 0.01 & 0.02 & 0.01 \\
\hline & Before & $0.52^{*}$ & 0.62 & 0.64 \\
\hline Low light + Snort & After & $0.50^{*}$ & 0.60 & 0.63 \\
\hline & Difference & 0.02 & 0.02 & 0.01 \\
\hline & Before & 0.58 & 0.61 & 0.65 \\
\hline High UV & After & 0.54 & 0.60 & 0.64 \\
\hline & Difference & 0.04 & 0.01 & 0.01 \\
\hline & Before & $0.51^{*}$ & 0.55 & 0.66 \\
\hline Low lignt + Hign & After & $0.50^{*}$ & $0.54^{*}$ & 0.65 \\
\hline & Difference & 0.01 & 0.01 & 0.01 \\
\hline L.S.D a & $\leq 0.05$ & 0.06 & 0.07 & 0.05 \\
\hline
\end{tabular}


Younis, M. E. et al.

Table5: The effect of low light and UV radiations either alone or in combination on water content $\left(\mathrm{g}\right.$ seedling $\left.^{-1}\right)$ of Vicia faba seedlings germinated under dark conditions. Mean values are significantly different from control at ${ }^{*} \mathrm{P} \leq \mathbf{0 . 0 5}$.

\begin{tabular}{|c|c|c|c|c|}
\hline \multicolumn{2}{|c|}{ Treatment Day } & 2nd & 4th & 6th \\
\hline \multicolumn{2}{|c|}{ Control (dark) } & 1.95 & 2.04 & 2.1 \\
\hline \multirow{3}{*}{ Low light } & Before & $1.89^{*}$ & $1.92^{*}$ & 2.01 \\
\hline & After & $1.87^{*}$ & $1.89^{*}$ & 1.99 \\
\hline & Difference & 0.02 & 0.03 & 0.02 \\
\hline \multirow{3}{*}{ Short UV } & Before & 1.96 & 2.02 & 2.00 \\
\hline & After & 1.90 & 1.99 & $1.96^{*}$ \\
\hline & Difference & 0.06 & 0.03 & 0.04 \\
\hline \multirow{3}{*}{$\underset{\text { UV }}{\text { Low light }+ \text { Short }}$} & Before & 2.01 & 2.02 & 2.09 \\
\hline & After & $1.88^{*}$ & 1.95 & 2.05 \\
\hline & Difference & 0.13 & 0.07 & 0.04 \\
\hline \multirow{3}{*}{ High UV } & Before & 1.94 & 1.95 & 2.01 \\
\hline & After & $1.89^{*}$ & 1.95 & 2.01 \\
\hline & Difference & 0.05 & 0.00 & 0.00 \\
\hline \multirow{3}{*}{$\underset{\text { UV }}{\text { Low light }+ \text { High }}$} & Before & $1.88^{*}$ & $1.88^{*}$ & $1.92^{*}$ \\
\hline & After & $1.86^{*}$ & $1.87^{*}$ & $1.92^{*}$ \\
\hline & Difference & 0.02 & 0.01 & 0.00 \\
\hline \multicolumn{2}{|c|}{ L.S.D at ${ }^{*} \mathrm{P} \leq 0.05$} & 0.05 & 0.11 & 0.12 \\
\hline
\end{tabular}

Table6: The effect of high light and UV radiations either alone or in combination on the length of radicle $\left(\mathrm{cm}\right.$ seedling ${ }^{-1}$ ) of Vicia faba seedlings germinated under light conditions. Mean values are significantly different from control at ${ }^{*} \mathrm{P} \leq \mathbf{0 . 0 5}$.

\begin{tabular}{|c|c|c|c|c|}
\hline Treatment & Day & 2nd & 4th & 6th \\
\hline Con & ght) & 4.56 & 5.28 & 5.40 \\
\hline & Before & 4.55 & $4.73^{*}$ & $4.85^{\star}$ \\
\hline High light & After & 4.53 & $4.70^{*}$ & $4.75^{\star}$ \\
\hline & Difference & 0.02 & 0.03 & 0.10 \\
\hline & Before & 4.50 & $4.56^{*}$ & $4.70^{*}$ \\
\hline Short UV & After & 4.40 & $4.46^{\star}$ & $4.63^{*}$ \\
\hline & Difference & 0.10 & 0.10 & 0.07 \\
\hline & Before & 4.65 & $4.74^{*}$ & $4.85^{\star}$ \\
\hline High light + Short & After & 4.50 & $4.55^{\star}$ & $4.80^{*}$ \\
\hline & Difference & 0.15 & 0.19 & 0.05 \\
\hline L.S.D & 0.05 & 0.22 & 0.26 & 0.27 \\
\hline
\end{tabular}

Table7: The effect of high light and UV radiations either alone or in combination on the length of plumule $\left(\mathrm{cm}\right.$ seedling $\left.{ }^{-1}\right)$ of Vicia faba seedlings germinated under light conditions. Mean values are significantly different from control at ${ }^{*} P \leq 0.05$.

\begin{tabular}{|c|c|c|c|c|}
\hline \multicolumn{2}{|c|}{ Day } & 2nd & 4th & 6th \\
\hline \multirow{3}{*}{ Control (light) } & 5.28 & 6.50 & 7.20 \\
\hline \multirow{3}{*}{ High light } & Before & 5.20 & 6.55 & $6.90^{*}$ \\
\cline { 2 - 5 } & After & 5.10 & 6.30 & $6.80^{*}$ \\
\cline { 2 - 5 } & Difference & 0.10 & 0.25 & 0.10 \\
\hline \multirow{3}{*}{ Short UV } & Before & $5.00^{*}$ & $6.00^{*}$ & 7.20 \\
\cline { 2 - 5 } & After & 5.20 & $5.80^{*}$ & 7.10 \\
\cline { 2 - 5 } & Difference & 0.20 & 0.20 & 0.10 \\
\hline \multirow{3}{*}{$\begin{array}{c}\text { High light + Short } \\
\text { UV }\end{array}$} & Before & 5.35 & $5.95^{*}$ & $6.20^{*}$ \\
\cline { 2 - 5 } & After & 5.25 & $5.80^{*}$ & $6.10^{*}$ \\
\cline { 2 - 5 } & Difference & 0.10 & 0.15 & 0.10 \\
\hline \multicolumn{2}{|r|}{ L.S.D at ${ }^{*} \mathbf{P} \leq \mathbf{0 . 0 5}$} & 0.21 & 0.26 & 0.27 \\
\hline
\end{tabular}


Table8: The effect of high light and UV radiations either alone or in combination on fresh mass ( $\mathrm{g}_{\text {seedling }}{ }^{-1}$ ) of Vicia faba seedlings germinated under light conditions. Mean values are significantly different from control at ${ }^{*} \mathrm{P} \leq \mathbf{0 . 0 5}$.

\begin{tabular}{|c|c|c|c|c|}
\hline Treatment Day & 2nd & 4th & 6th \\
\hline \multicolumn{2}{|c|}{ Control (light) } & 2.62 & 2.74 & 2.89 \\
\hline \multirow{3}{*}{ High light } & Before & 2.61 & 2.64 & 2.79 \\
\cline { 2 - 5 } & After & $2.46^{*}$ & $2.57^{*}$ & 2.77 \\
\cline { 2 - 5 } & Difference & 0.15 & 0.07 & 0.02 \\
\hline \multirow{3}{*}{ Short UV } & Before & 2.68 & 2.70 & $2.74^{*}$ \\
\cline { 2 - 5 } & After & 2.52 & 2.60 & $2.72^{*}$ \\
\cline { 2 - 5 } High light + & Difference & 0.16 & 0.1 & 0.02 \\
\cline { 2 - 5 } Short UV & Before & 2.62 & 2.66 & $2.74^{*}$ \\
\cline { 2 - 5 } & After & $2.48^{*}$ & 2.61 & $2.70^{*}$ \\
\hline \multicolumn{2}{|r}{} & 0.14 & 0.05 & 0.04 \\
\hline \multirow{2}{*}{ L.S.D at ${ }^{*} \mathbf{P} \leq \mathbf{0 . 0 5}$} & 0.13 & 0.14 & 0.14 \\
\hline
\end{tabular}

Table9: The effect of high light and UV radiations either alone or in combination on dry mass ( $\mathrm{g}$ seedling ${ }^{-1}$ ) of Vicia faba seedlings germinated under light conditions. Mean values are significantly different from control at ${ }^{*} \mathrm{P} \leq 0.05$.

\begin{tabular}{|c|c|c|c|c|}
\hline \multicolumn{2}{|c|}{ Treatment Day } & 2nd & 4th & 6th \\
\hline \multicolumn{2}{|c|}{ Control (light) } & 1.98 & 2.08 & 2.18 \\
\hline \multirow{3}{*}{ High light } & Before & 1.98 & 2.00 & 2.11 \\
\hline & After & $1.86^{*}$ & $1.95^{\star}$ & 2.10 \\
\hline & Difference & 0.12 & 0.05 & 0.01 \\
\hline \multirow{3}{*}{ Short UV } & Before & 2.01 & 2.04 & $2.05^{\star}$ \\
\hline & After & $1.90^{*}$ & $1.97^{*}$ & $2.05^{*}$ \\
\hline & Difference & 0.11 & 0.07 & 0.00 \\
\hline \multirow{3}{*}{$\begin{array}{c}\text { High light }+ \text { Short } \\
\text { UV }\end{array}$} & Before & 1.98 & 2.01 & $2.05^{*}$ \\
\hline & After & $1.88^{*}$ & 1.98 & $2.04^{*}$ \\
\hline & Difference & 0.13 & 0.03 & 0.01 \\
\hline \multicolumn{2}{|c|}{ L.S.D at ${ }^{*} P \leq 0.05$} & 0.05 & 0.10 & 0.11 \\
\hline
\end{tabular}

Table10: The effect of high light and UV radiations either alone or in combination on water contnet $\left(g\right.$ seedling $\left.^{-1}\right)$ of Vicia faba seedlings germinated under light conditions. Mean values are significantly different from control at ${ }^{*} \mathrm{P} \leq 0.05$.

\begin{tabular}{|c|c|c|c|c|}
\hline \multirow{2}{*}{\multicolumn{2}{|c|}{\begin{tabular}{|cc} 
Treatment & Day \\
Control (light) &
\end{tabular}}} & 2nd & 4th & 6th \\
\hline & & 0.64 & 0.66 & 0.71 \\
\hline \multirow{3}{*}{ High light } & Before & 0.63 & 0.64 & 0.69 \\
\hline & After & $0.60^{*}$ & $0.62^{*}$ & $0.66^{*}$ \\
\hline & Difference & 0.03 & 0.02 & 0.03 \\
\hline \multirow{3}{*}{ Short UV } & Before & 0.67 & 0.66 & 0.69 \\
\hline & After & 0.62 & $0.63^{*}$ & $0.67^{*}$ \\
\hline & Difference & 0.05 & 0.03 & 0.02 \\
\hline \multirow{3}{*}{$\begin{array}{c}\text { High light }+ \text { Short } \\
\text { UV }\end{array}$} & Before & 0.64 & 0.65 & 0.69 \\
\hline & After & $0.60^{*}$ & $0.62^{*}$ & $0.66^{*}$ \\
\hline & Difference & 0.04 & 0.02 & 0.03 \\
\hline \multicolumn{2}{|l|}{ L.S.D at ${ }^{*} P \leq$} & 0.03 & 0.02 & 0.03 \\
\hline
\end{tabular}


Decreased growth parameters (length of radicle, length of plumule, fresh mass, dry mass and water content) of broad bean seedlings over a period of 6 days, in the present study are likely the result of lower rates of $\mathrm{CO}_{2}$ assimilation in seedlings germinated with UV-radiation. Changes in biomass enhanced by UV radiations which was observed in the broad bean seedlings under investigation may increase their environmental stress tolerance. Changes in plant height often occurs in conjunction with change in stem diameter and self-shading by foliage, which reduces heat load at the base of the seedlings and minimizes cellular damage that occurs at high surface soil treatments (Helgerson, 1990).

Changes in photosynthetic capacity:

White light (low and high intensity) and UV-A or UV-C irradiation either alone or in combination treatment resulted in the reduction of the synthesis of chloroplast pigments ( $\mathrm{Chl} \mathrm{a,} \mathrm{Chl} \mathrm{b} \mathrm{and} \mathrm{carotenoids)} \mathrm{in} \mathrm{broad}$ beans seedlings. All treatments resulted in a reduction in chlorophyll content (tables 11,12). The $\mathrm{Chl} a / b$ ratio, $\mathrm{Chl} a+b$ and total pigments content of the differently treated broad bean seedlings visibly changed with the treatment in light intensity and UV irradiation (tables 11,12).

Photosynthetic capacity (PS II) was significantly and variably decreased in broad bean seedlings treated with rather white light, UV-A or UV-C irradiation either alone or in combination, as compared with control seedlings germinated either in dark or in light (tables 13,14).

Photosynthetic pigments, mainly constitute of chlorophyll a, chlorophyll b and carotenoids, are of vital importance in photosynthesis. Great reductions in photosynthetic pigments were observed in broad bean seedlings treated with white light and/ or UV-A and UV-C irradiations. Pigments of the photosynthetic apparatus can be destroyed by UV irradiation, with concomitant lass of photosynthetic capacity (Jordan et al., 1994, Michaela et al., 2000; Laposi et al., 2002; Saleh et al., 2006).

Chlorophylls and carotenoids may be adversely affected by relatively large amounts of UV-b and UV-c radiation, where carotenoids are generally being less affected than chlorophylls (Pfundel et al., 1992). It has been reported that UV-B and UV-C radiation resulted in greater reduction in the amount of $\mathrm{Chl} \mathrm{b}$ as opposed to $\mathrm{Chl}$ a and might point a more selective destruction of $\mathrm{Chl} b$ biosynthesis or degradation of precursors (Marwood and Greeberg, 1996).

Saleh et al. (2006) stated that the reduction in carbohydrate contents of broad bean seedlings, in response to elevated UV radiation could be attributed to the destructive damage of photosystems induced by UV radiation, which led to the decrease in photosynthetic efficiency. UV-A and UV-B induced inhibition of photosynthesis in many plant species. It is evident that UV radiation can potentially impair the performance of main component processes of photosynthesis, the photophosphorylation reactions of the thylakoid membrane, the $\mathrm{CO}_{2}$-fixation reactions of the Calvin cycle and stomatal control of $\mathrm{CO}_{2}$ supply (Allen et al., 1998). 
Table 11: The effect of low light and UV radiations either alone or in combination on pigments ( $\mu \mathrm{g} / 100 \mathrm{~g}$ fresh mass) of Vicia faba seedlings germinated under dark conditions. Mean values are significantly different from control at ${ }^{*} P \leq 0.05$

\begin{tabular}{|c|c|c|c|c|c|c|c|c|}
\hline Day & \multicolumn{2}{|c|}{ Treatment } & Chl a & Chl b & Cars & Chl $\mathbf{a}+\mathbf{b}$ & Chl a / b & $\begin{array}{c}\text { Total } \\
\text { pigments }\end{array}$ \\
\hline \multirow{17}{*}{ ํㅗํ } & \multicolumn{2}{|c|}{ Control (dark) } & 120 & 330 & 270 & 450 & 0.36 & 720.0 \\
\hline & \multirow{3}{*}{ Low light } & Before & 117.6 & 345.4 & 263.7 & 463 & 0.34 & 726.7 \\
\hline & & After & $66.6^{*}$ & $91.1^{*}$ & 289.1 & $157.7^{\star}$ & 0.73 & $446.8^{*}$ \\
\hline & & Difference & 51 & 254.3 & -25.4 & 305.3 & -0.39 & 279.9 \\
\hline & \multirow{3}{*}{ Short UV } & Before & 114.6 & 320.3 & $220.5^{*}$ & $434.9^{*}$ & 0.35 & $655.4^{*}$ \\
\hline & & After & $84.4^{*}$ & $242.4^{*}$ & $172.2^{*}$ & $326.8^{*}$ & 0.35 & 499.0* \\
\hline & & Difference & 30.2 & 77.9 & 48.3 & 108.1 & 0.00 & 156.4 \\
\hline & \multirow{3}{*}{$\begin{array}{c}\begin{array}{c}\text { Low light } \\
+ \text { short } \\
\text { UV }\end{array} \\
\end{array}$} & Before & 115.7 & 334.6 & $250.0^{*}$ & 450.3 & 0.34 & $700.3^{*}$ \\
\hline & & After & $100.5^{*}$ & $292.8^{*}$ & $195.2^{*}$ & 393.3 & 0.34 & $588.5^{\star}$ \\
\hline & & Difference & 15.2 & 41.8 & 54.8 & 57.0 & 0.00 & 11.8 \\
\hline & \multirow{3}{*}{ Long UV } & Before & $150.7^{\star}$ & $313.1^{*}$ & $237.0^{*}$ & 463.8 & 0.48 & $700.8^{*}$ \\
\hline & & After & $107.3^{*}$ & $285.4^{*}$ & $196.7^{*}$ & $392.7^{*}$ & 0.37 & $589.4^{\star}$ \\
\hline & & Difference & 43.4 & 27.7 & 40.3 & 71.1 & 0.11 & 111.4 \\
\hline & \multirow{3}{*}{$\begin{array}{c}\text { Low light } \\
+ \text { long } \\
\text { UV }\end{array}$} & Before & 137.4 & 345.7 & $246.7^{*}$ & 483.1 & 0.40 & 729.8 \\
\hline & & After & $102.8^{*}$ & $249.1^{*}$ & $214.5^{*}$ & $351.9^{*}$ & 0.40 & $566.4^{*}$ \\
\hline & & Difference & 34.6 & 96.6 & 32.2 & 131.2 & 0.00 & 163.4 \\
\hline & \multicolumn{2}{|c|}{ L.S.D at ${ }^{*} P \leq 0.05$} & 7.6 & 11.8 & 8.7 & 11.5 & 0.01 & 14.5 \\
\hline \multirow{17}{*}{$\begin{array}{l}\frac{\pi}{0} \\
\dot{y}\end{array}$} & \multicolumn{2}{|c|}{ Control (dark) } & 238.0 & 519.9 & 352.3 & 757.9 & 0.45 & 1110.2 \\
\hline & \multirow{3}{*}{ Low light } & Before & 194.0* & $107.9^{*}$ & $307.7^{*}$ & $301.9^{*}$ & 1.80 & $609.6^{\star}$ \\
\hline & & After & $163.3^{*}$ & $84.2^{*}$ & $272.7^{*}$ & $247.5^{*}$ & 1.94 & $520.2^{*}$ \\
\hline & & Difference & 30.7 & 23.7 & 35.0 & 54.4 & -0.14 & 89.4 \\
\hline & \multirow{3}{*}{ Short UV } & Before & $96.7^{*}$ & $248.7^{*}$ & $180.6^{*}$ & $345.4^{*}$ & $0.39^{*}$ & $526.0^{*}$ \\
\hline & & After & $72.3^{*}$ & $165.8^{*}$ & $160.2^{*}$ & $238.1^{*}$ & $0.43^{*}$ & 398.3* \\
\hline & & Difference & 24.4 & 82.9 & 20.4 & 107.3 & -0.04 & 127.7 \\
\hline & \multirow{3}{*}{\begin{tabular}{|c|}
$\begin{array}{c}\text { Low light } \\
+ \\
\text { short } \\
\text { UV }\end{array}$ \\
\end{tabular}} & Before & $120.2^{*}$ & $296.1^{*}$ & 199.9* & $416.3^{*}$ & $0.41^{*}$ & $616.2^{*}$ \\
\hline & & After & $101.4^{*}$ & $276.0^{*}$ & $137.2^{*}$ & $377.4^{*}$ & $0.37^{*}$ & $514.6^{*}$ \\
\hline & & Difference & 18.8 & 20.1 & 62.7 & 38.9 & 0.04 & 101.6 \\
\hline & \multirow{3}{*}{ Long UV } & Before & $110.1^{*}$ & $293.3^{*}$ & $198.7^{*}$ & $403.4^{*}$ & $0.38^{*}$ & $602.1^{*}$ \\
\hline & & After & $86.2^{*}$ & $270.0^{*}$ & $167.6^{*}$ & $356.2^{*}$ & $0.32^{*}$ & $523.8^{*}$ \\
\hline & & Difference & 23.9 & 23.3 & 31.1 & 47.2 & 0.06 & 78.3 \\
\hline & \multirow{3}{*}{$\begin{array}{c}\text { Low light } \\
+ \text { long } \\
\text { UV }\end{array}$} & Before & $110.6^{*}$ & $256.1^{*}$ & $220.6^{*}$ & $366.7^{*}$ & $0.43^{*}$ & $587.3^{*}$ \\
\hline & & After & $91.6^{*}$ & $237.2^{*}$ & $209.1^{*}$ & $328.8^{*}$ & $0.39^{*}$ & $537.9^{*}$ \\
\hline & & Difference & 19 & 18.9 & 11.5 & 37.9 & 0.04 & 49.4 \\
\hline & \multicolumn{2}{|c|}{ L.S.D at ${ }^{*} P \leq 0.05$} & 11.6 & 21.3 & 16.4 & 17.6 & 0.01 & 19.3 \\
\hline \multirow{17}{*}{$\begin{array}{l}\frac{\pi}{0} \\
\frac{0}{0}\end{array}$} & \multicolumn{2}{|c|}{ Control (dark) } & 331.9 & 661.3 & 402.9 & 993.2 & 0.50 & 1396.1 \\
\hline & & Before & $170.6^{*}$ & $94.3^{*}$ & $275.6^{*}$ & $264.9^{*}$ & 1.81 & $540.5^{*}$ \\
\hline & Low light & After & $131.2^{*}$ & $70.1^{*}$ & $251.7^{*}$ & $201.3^{*}$ & 1.87 & $453.0^{*}$ \\
\hline & & Difference & 39.4 & 24.2 & 23.9 & 63.6 & -0.06 & 87.5 \\
\hline & & Before & $80.6^{*}$ & $170.2^{*}$ & $166.7^{*}$ & $250.8^{*}$ & $0.47^{*}$ & $417.5^{*}$ \\
\hline & Short UV & After & $61.2^{*}$ & $141.2^{*}$ & $137.8^{*}$ & $202.4^{*}$ & $0.43^{*}$ & $340.2^{\star}$ \\
\hline & & Difference & 19.4 & 29.0 & 28.9 & 48.4 & 0.04 & 77.3 \\
\hline & Low light & Before & $109.6^{*}$ & $279.3^{*}$ & $201.7^{*}$ & $388.9^{*}$ & $0.39^{*}$ & $590.6^{*}$ \\
\hline & + short & After & $82.7^{*}$ & $243.1^{*}$ & $174.0^{*}$ & $325.8^{*}$ & $0.34^{*}$ & $472.8^{*}$ \\
\hline & & Difference & 26.9 & 36.2 & 27.7 & 63.1 & 0.05 & 117.8 \\
\hline & & Before & $90.3^{*}$ & $268.0^{*}$ & $171.2^{*}$ & $358.3^{*}$ & $0.34^{*}$ & $529.5^{*}$ \\
\hline & Long UV & After & $72.1^{*}$ & $241.4^{*}$ & $134.2^{*}$ & $313.5^{*}$ & $0.30^{*}$ & $447.7^{\star}$ \\
\hline & & Difference & 18.2 & 26.6 & 37.0 & 44.8 & 0.04 & 81.8 \\
\hline & Low light & Before & $69.2^{*}$ & $241.3^{*}$ & $212.6^{*}$ & $310.5^{*}$ & $0.30^{*}$ & $523.1^{*}$ \\
\hline & + long & After & $66.2^{*}$ & $216.2^{*}$ & $176.3^{*}$ & $282.8^{*}$ & $0.30^{*}$ & $459.1^{\star}$ \\
\hline & UV & Difference & 3.0 & 25.1 & 36.3 & 27.7 & 0.00 & 64.0 \\
\hline & L.S.D a & ${ }^{*} P \leq 0.05$ & 16.4 & 22.2 & 18.3 & 19.0 & 0.02 & 19.6 \\
\hline
\end{tabular}


Younis, M. E. et al.

Table 12: The effect of high light and UV radiations either alone or in combination on pigments ( $\mu \mathrm{g} / 100 \mathrm{~g}$ fresh mass) of Vicia faba seedlings germinated under light conditions. Mean values are significantly different from control at ${ }^{*} \mathrm{P} \leq \mathbf{0 . 0 5}$.

\begin{tabular}{|c|c|c|c|c|c|c|c|c|}
\hline Day & \multicolumn{2}{|c|}{ Treatment } & Chl a & Chl b & Cars & Chl $a+b$ & Chl a / b & $\begin{array}{c}\text { Total } \\
\text { pigments }\end{array}$ \\
\hline \multirow{11}{*}{ 을 } & \multicolumn{2}{|c|}{ Control (light) } & 543.6 & 427.9 & 339.3 & 971.5 & 1.27 & 1310.8 \\
\hline & \multirow{3}{*}{ High light } & Before & 536.7 & 420.6 & $310.6^{*}$ & 957.3 & 1.28 & $1267.9^{*}$ \\
\hline & & After & $421.9^{*}$ & $305.8^{*}$ & $281.6^{*}$ & $727.7^{*}$ & 1.38 & $1009.3^{*}$ \\
\hline & & Difference & 114.8 & 114.8 & 29.0 & 229.6 & -0.10 & 258.6 \\
\hline & \multirow{3}{*}{ Short UV } & Before & $514.4^{*}$ & 434.3 & $313.0^{*}$ & $948.7^{*}$ & $1.18^{*}$ & $1261.7^{*}$ \\
\hline & & After & $481.2^{*}$ & $374.5^{*}$ & $296.3^{*}$ & $855.7^{*}$ & 1.28 & $1152^{*}$ \\
\hline & & Difference & 33.2 & 59.8 & 16.7 & 93.0 & -0.10 & 109.7 \\
\hline & \multirow{3}{*}{$\begin{array}{l}\text { High light+ } \\
\text { Short UV }\end{array}$} & Before & $531.8^{*}$ & 434.8 & $322.6^{*}$ & 966.6 & $1.22^{*}$ & $1289.2^{*}$ \\
\hline & & After & $351.2^{*}$ & $333.7^{*}$ & $262.2^{*}$ & $684.9^{*}$ & $1.05^{*}$ & $947.1^{*}$ \\
\hline & & Difference & 180.6 & 101.1 & 60.4 & 281.7 & 0.17 & 342.1 \\
\hline & L.S.D at & ${ }^{\star} \mathrm{P} \leq 0.05$ & 9.8 & 8.1 & 8.9 & 20.6 & 0.03 & 21.7 \\
\hline \multirow{11}{*}{$\begin{array}{l}\vec{\sigma} \\
\text { ठ } \\
\text { J }\end{array}$} & \multicolumn{2}{|c|}{ Control (light) } & 607.3 & 485.5 & 496.7 & 1092.8 & 1.25 & 1589.5 \\
\hline & \multirow{3}{*}{ High light } & Before & $424.3^{*}$ & $373.4^{*}$ & $315.4^{*}$ & $797.7^{*}$ & $1.14^{*}$ & $1113.1^{*}$ \\
\hline & & After & $251.5^{\star}$ & $306.8^{*}$ & $202.2^{*}$ & $558.3^{*}$ & $0.82^{*}$ & $760.5^{*}$ \\
\hline & & Difference & 172.8 & 66.6 & 113.2 & 239.4 & 0.32 & 352.6 \\
\hline & \multirow{3}{*}{ Short UV } & Before & $490.1^{*}$ & $380.6^{*}$ & $301.8^{*}$ & $870.7^{*}$ & 1.29 & $1172.5^{\star}$ \\
\hline & & After & $457.3^{*}$ & $360.4^{*}$ & $266.1^{*}$ & $817.7^{*}$ & 1.27 & $1083.8^{*}$ \\
\hline & & Difference & 32.8 & 20.2 & 35.7 & 53.0 & 0.02 & 88.7 \\
\hline & \multirow{3}{*}{$\begin{array}{l}\text { High light+ } \\
\text { Short UV }\end{array}$} & Before & $350.0^{*}$ & $325.6^{*}$ & $271.6^{*}$ & $675.6^{*}$ & $1.07^{*}$ & $947.2^{*}$ \\
\hline & & After & $311.2^{*}$ & $303.6^{*}$ & $248.6^{\star}$ & $614.8^{*}$ & $1.03^{*}$ & $863.4^{*}$ \\
\hline & & Difference & 38.8 & 22.0 & 23.0 & 61.2 & 0.04 & 83.8 \\
\hline & L.S.D at & ${ }^{\star} P \leq 0.05$ & 11.5 & 9.9 & 10.1 & 18.3 & 0.05 & 17.2 \\
\hline \multirow{11}{*}{$\begin{array}{l}\overrightarrow{0} \\
\overline{0} \\
\overline{0}\end{array}$} & \multicolumn{2}{|c|}{ Control (light) } & 675.7 & 566.4 & 520.6 & 1242.1 & 1.19 & 1762.7 \\
\hline & \multirow{3}{*}{ High light } & Before & $270.0^{*}$ & $320.0^{*}$ & $290.6^{*}$ & $590.0^{*}$ & $0.84^{*}$ & $880.6^{*}$ \\
\hline & & After & $241.0^{*}$ & $292.0^{*}$ & $261.6^{*}$ & $533.0^{*}$ & $0.83^{*}$ & $794.6^{*}$ \\
\hline & & \begin{tabular}{|l|} 
Difference \\
\end{tabular} & 29.0 & 28.0 & 29.0 & 57.0 & 0.01 & 86.0 \\
\hline & \multirow{3}{*}{ Short UV } & Before & $461.7^{*}$ & $361.1^{*}$ & $270.8^{*}$ & $822.8^{*}$ & 1.28 & $1093.6^{*}$ \\
\hline & & After & $432.3^{*}$ & $336.2^{*}$ & $246.3^{*}$ & $768.5^{*}$ & 1.28 & $1014.8^{*}$ \\
\hline & & Difference & 29.4 & 24.9 & 24.5 & 54.3 & 0.00 & 78.8 \\
\hline & \multirow{3}{*}{$\begin{array}{l}\text { High light+ } \\
\text { Short UV }\end{array}$} & Before & $315.6^{*}$ & $306.7^{*}$ & $256.7^{*}$ & $622.3^{*}$ & $1.03^{*}$ & $879.0^{*}$ \\
\hline & & After & $281.9^{*}$ & $272.1^{*}$ & $228.9^{*}$ & $554.0^{*}$ & $1.03^{*}$ & $782.9^{*}$ \\
\hline & & Difference & 33.7 & 34.6 & 27.8 & 68.3 & 0.00 & 96.1 \\
\hline & \multicolumn{2}{|c|}{ L.S.D at ${ }^{*} P \leq 0.05$} & 14.7 & 12.8 & 11.6 & 21.2 & 0.02 & 22.1 \\
\hline
\end{tabular}

Table 13: The effect of high light and UV radiations either alone or in combination on PS (II) activity ( $\mu$ M DCPIP reduced $/ 100 \mathrm{mg}$ $\mathrm{Chl} / \mathrm{h}$ ) of Vicia faba seedlings germinated under light conditions. Mean values are significantly different from control at ${ }^{*} \mathrm{P} \leq 0.05$.

\begin{tabular}{|c|c|c|c|c|}
\hline \multicolumn{2}{|c|}{ Treatment } & 2nd & 4th & 6th \\
\hline \multicolumn{2}{|c|}{ Control (light) } & 8.4 & 15.9 & 21.2 \\
\hline \multirow{2}{*}{ High light } & Before & 8.3 & $15.0^{*}$ & 21.1 \\
\cline { 2 - 5 } & After & $6.7^{*}$ & $11.8^{*}$ & $15.6^{*}$ \\
\hline \multirow{2}{*}{ Short UV } & Before & 8.8 & $15.0^{*}$ & $20.9^{*}$ \\
\cline { 2 - 5 } & After & $5.4^{*}$ & $13.8^{*}$ & $17.5^{*}$ \\
\hline \multirow{2}{*}{ High light+ Short } & Before & 8.1 & $14.6^{*}$ & 21.0 \\
\cline { 2 - 5 } UV & After & $4.1^{*}$ & $10.7^{*}$ & $15.3^{*}$ \\
\hline \multicolumn{2}{|c|}{ L.S.D at ${ }^{*} \mathbf{P} \leq \mathbf{0 . 0 5}$} & 0.21 & 0.71 & 0.92 \\
\hline
\end{tabular}


Table 14: The effect of low light and UV radiations either alone or in combination on PS (II) activity ( $\mu$ M DCPIP reduced $/ 100 \mathrm{mg}$ $\mathrm{Chl} / \mathrm{h}$ ) of Vicia faba seedlings germinated under dark conditions. Mean values are significantly different from control at ${ }^{*} \mathrm{P} \leq 0.05$.

\begin{tabular}{|c|c|c|c|c|}
\hline \multicolumn{2}{|c|}{ Treatment Day } & 2nd & 4th & 6th \\
\hline \multicolumn{2}{|c|}{\begin{tabular}{|c|} 
Control (dark) \\
\end{tabular}} & 4.1 & 5.7 & 7.3 \\
\hline \multirow{2}{*}{ Low light } & Before & 4.3 & $5.2^{*}$ & $6.9^{*}$ \\
\hline & After & $3.1^{*}$ & $4.1^{*}$ & $4.8^{*}$ \\
\hline \multirow{2}{*}{ Short UV } & Before & 4.4 & 5.8 & 7.0 \\
\hline & After & $3.7^{*}$ & $3.9^{*}$ & $4.2^{*}$ \\
\hline \multirow{2}{*}{$\begin{array}{c}\text { Low light + short } \\
\text { UV }\end{array}$} & Before & 4.6 & 5.5 & $6.3^{*}$ \\
\hline & After & $2.2^{*}$ & $3.1^{*}$ & $3.9^{*}$ \\
\hline \multirow{2}{*}{ Long UV } & Before & 4.5 & 5.7 & 7.1 \\
\hline & After & $2.1^{*}$ & $2.7^{*}$ & $3.6^{*}$ \\
\hline \multirow{2}{*}{ Low light + long UV } & Before & 4.2 & 5.6 & $6.8^{*}$ \\
\hline & After & $1.2^{*}$ & $2.1^{\star}$ & $2.8^{*}$ \\
\hline \multicolumn{2}{|c|}{ L.S.D at ${ }^{*} P \leq 0.05$} & 0.2 & 0.25 & 0.35 \\
\hline
\end{tabular}

Difference in chlorophyll biosynthesis capacity was seen when epicotyls of dark-grown pea was irradiated (Virgin, 1993), and in light-grown seedlings of pine, where the chlorophyll content decreased downwards the seedlings (Spano et al., 1992). Chlorophyll formation capacity along the bean seedlings was correlated to the amount of protchlorophyllide present before irradiation (Mc Ewen et al., 1996). The amount of protchlorophyllide decreased downwards in dark-grown seedlings as did the amount of chlorophyll formed after irradiation. Furthermore, protchlorophyllide regarded as the main phototransformable form, diminished downwards the seedlings.

In nature the hypocotyls will normally extend and reach light some days after germination. Several processes will start when the hook reads light ( Mc Ewen et al., 1996). The seedlings will change from an etiolated way of growing to photomorphogenic development. Chlorophyll biosynthesis in the hook section and the upper parts of the hypocotyls can presumably contribute to an early production of photosynthetic products.

\section{REFERENCES}

Allen, D.J., Nogues, S. and Baker, N.R. (1998). Ozone depletion and increased UV-B radiation: is there a real threat to photosynthesis. $\mathrm{J}$. Exp. Bot. 49: (328):1775-1788.

Arnon, D. I. (1949) Copper enzyme in isolated chloroplasts. Polyphenol axidase in Beta vulgaris. Plant Physiol. 24, 1-15.

Caldwell, M.M., Ballare, C.L., Bornman, J.F., Flint, S.D., Bjorn, L.O., Teramura, A.H., Kulandaivelu, G. and Tevini, M. (2003). Terrestrial ecosystems, increased solar ultraviolet radiation and interactions with other climatic change factors. Photochem. Photobiol. Sci.,2: 29-38.

Demir, Y. (2000). Growth and proline content of germinating wheat genotypes under ultraviolet light. Turk. J. Bot. 24: 67-70. 


\section{Younis, M. E. et al.}

Farman, J.C., Gardiner, B.G., and Shanklin, J.D. (1985). Large losses of ozone in Antarctica reveal seasonal CIO/NO interation. Nature. 315: 207-210

Gehrke, C., (1999). Impacts of Enhanced Ultraviolet-B Radiation on Mosses in a Subarctic Health Ecosystem. Ecology. http://www.findarticles.com/p/articles/mi_m2120/is_6_80/ai_56022611. $\mathrm{html}$.

Gilbert, D. (1996). Osram Sylvania $\AA 350 B$ L Safety product safety data sheet, PSDS No. 1.1.4, fluorescent backlight lamps and Research and Development Manual.

Helgerson, O.T. (1990). Heat damage in tree seedling and its prevention. New Forests, 3: 333-358.

Jordan, B.R. (1996). The effects of UV-B radiation on plants: a molecular prospective. In: Callow J.A., ed. Advances in Botanical Research. Academic Press, 97-162.

Jordan, B.R., James, P.E., Strid, A. and Anthony, R.G. (1994). The effect of ultraviolet-B radiation on gene expression and pigment composition in etiolated and green pea leaf tissue UV-B induced changes are genespecific and dependent upon the developmental stage, Plant Cell and Environment. 17: 45-54.

Krause, G.H., Schmude, C., Garden, H., Koroleva, O.Y. and Winter, K. (1999). Effects of solar ultraviolet radiation on the potential efficiency of photosystem II in leaves of tropical plants. Plant Physiol. 121: 13491358

Laposi, R., Veres, Sz., Mile, O. and Meszaros, I. (2002). Photosynthesisecophysiological properties of beech (Fagus sylvestris L.) under the exclusion of ambient UV-B radiation. Proceedings of the $7^{\text {th }}$ Hungarian Congress on Plant Physiology, Acta Biologia Szegediensis. 46: 243245.

Marwood, C.A. and Greeberg, B.M. (1996). Effect of supplementary UV-B radiation on chlorophyll synthesis and accumulation of photosystems during chloroplast development in Spirodela oligorrhiza. J. Photochem. And Photobiol. 64: 664-670.

Mc Ewen, B., Seyyedi, M., Younis, S. and Sundqvist, C. (1996). Formation of short-wavelength chlorophyllide after brief irradiation is correlated with the occurrence of protochlorophyllide in dark-grown epi-and hypocotyls of bean (Phaseolus vulgaris). Physiol. Plant. 96:51-58.

Metzner, H., Rau, H. and Senger, H. (1965). Untersuchungen Zur synchronixier Barklet Einzelner Pigment mangel. Multantent Von Chlorella Planta. 65: 186-199.

Michaela, A., Norbert, K. and George, N. (2000). Effect of Cold and UV-B stress on scavenging systems of Phaseolus vulgaris leaves. Poster. American Society of Plant Biologist. Found online at http://www.unibonn.de/obstbau.

Musil, C. F. (1996). Accumulated effect of elevated ultraviolet-B radiation over multiple generations of the arid-environment annual Dimorphothec sinuate DC. (Asteraceae). Plant Cell and Environment. 19(9): 10171027. 
Pfundel, E.E., PPan, R.S. and Dilley, R.A. (1992). Inhibition of violaxanthin deep oxidation by ultraviolet-B radiation in isolated chloroplasts and intact leaves. Plant physiol. 98:1372-1380.

Saleh, A.H., Abdel-Kader, D.Z. and Abu-Elsaoud, A.M. (2006). Metabolic responses of soybean (Glycine max) plant to increasing $U V(A+B)$ radiation. Assiut Univ. J. of Botany. 35(2): 107-125.

Saleh, A.H., Abdel-Kader, D.Z. and Abu-Elsaoud, A.M. (2006). Phenylpropanoid and Isopropanoid enhance tolerance to increased levels of $U V_{A+B}$ radiation in three cultivars of soybean (Glycine max) seedlings. J. Applied Sciences. 6(9): 1939-1953.

Saradhi, P., Alia, P., Sandeep, A., Prasad, K. and Arora, S. (1995). Proline accumulates in plants exposed to UV radiation and protects them against UV induced peroxidation. Biochemical and Biophysical Research Communications. 209(1) : 1-5.

Snedecor, W.; Cochron, G. (1980). Statistical Methods. Lowa State Univ. Press, Ames.

Spano, A. J., He, Z. and Timko, M. P. (1992). NADPH: protochlorophyllide oxidoreductases in white pine (Pinus strobes) and loblolly pine (Pinus taeda). Mol. Gen. Genet. 236:86-95.

Taylor, R.M., Tobin, A.K. and Bray, C.M. (1997). DNA damage and repair in plants. In: Lumsden P.J. (ed.) Plants and UV-B, Responses to Environmental Change. University press, Cambridge, pp. 53-76.

Todorov, D., Alexieva, V., Markov, V., Mapelli, S., and Kranov, E.(2003). Effect of UV-B-irradiation on growth some stress markers and enzymes of maize seedlings. Bulg. J. Plant Physiol., Special Issue 364-374

Virgin, H. I. (1993). Effectiveness of light of different wavelengths to induce chlorophyll biosynthesis in rapidly and slowly greening tissues. Physiol. Plant. 89: 761-766.

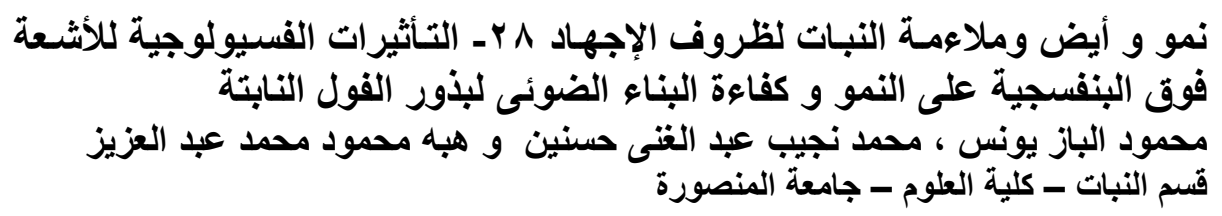

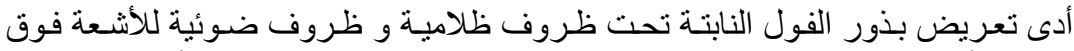

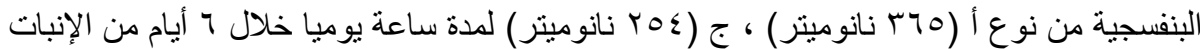

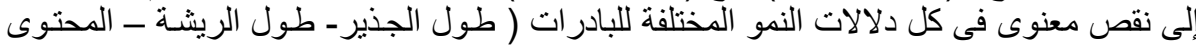

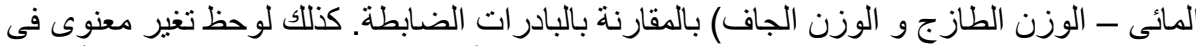

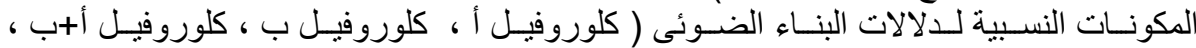

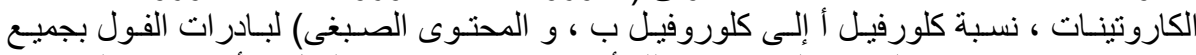

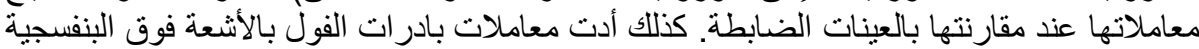

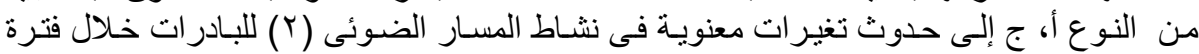

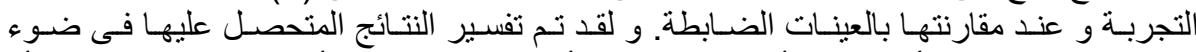
الميكانيكيات المنظمة لتأثير نمو و أيض البادرات بالأشعة فوق البنفسجية أثناء الإنبات في الظلام أو في 
Younis, M. E. et al. 